\title{
Local characterization of the optical properties of annealed Au films on glass substrates
}

\author{
R. Bernardo-Gavito ${ }^{1,2}$, A. Serrano ${ }^{3,4}$, M. A. García ${ }^{2,3}$, R. Miranda ${ }^{1,2,5}$, D. Granados ${ }^{2, a)}$ \\ ${ }^{1}$ Departamento de Física de la Materia Condensada, Universidad Autónoma de \\ Madrid,Cantoblanco 28049 Madrid, Spain \\ ${ }^{2}$ Instituto Madrileño de Estudios Avanzados en Nanociencia (IMDEA-Nanociencia),Cantoblanco \\ 28049 Madrid, Spain \\ ${ }^{3}$ Instituto de Cerámica y Vidrio ,Consejo Superior de Investigaciones Científicas, 28049 Madrid, \\ Spain \\ ${ }^{4}$ Departamento de Física de Materiales, Universidad Complutense de Madrid, 28040 Madrid, \\ Spain \\ 5Instituto de Ciencia de Materiales "Nicolas Cabrera", Universidad Autónoma de Madrid, \\ Cantoblanco 28049, Madrid, Spain
}

\begin{abstract}
We present scanning near field microscopy and local spectroscopic characterisation of gold nanoparticles fabricated on glass sodalime cover slides. The nanoislands are fabricated by the thermal annealing of gold thin films. Results are presented for samples annealed at $300^{\circ} \mathrm{C}$, $400^{\circ} \mathrm{C}$ and $500^{\circ} \mathrm{C}$. We study the spectral dependence of the transmittance at the nanoscale level with respect to the nanoislands size, shape and interparticle distance.
\end{abstract}

a) Corresponding autor. Electronic mail: daniel.granados@imdea.org 


\section{I - INTRODUCTION}

Surface Plasmon Resonance (SPR) is one of the most interesting properties of noble metals ${ }^{1,2,3}$. This collective oscillation of the conduction electrons can be optically excited, leading to a huge extinction cross section at the resonance frequency. Excitation of SPR allows to locally amplify and concentrate the light overcoming the diffraction limit ${ }^{4}$, rending noble metal nanoparticles (NPs) interesting for applications in a wide variety of fields ${ }^{5}$. In the case of Au NPs, their chemical stability as well as high biocompatibility and easy functionalisation, in combination with SPR yield the development of many applications for biomedical $^{6,7,8,9}$, environmental ${ }^{4,10,11}$, energy harvesting ${ }^{12,13,14}$ or optical purposes ${ }^{15,16}$.

A key limitation for the use of NPs in real applications is the fabrication of large quantities at affordable economical costs. Chemical synthesis or physical patterning of thin films can provide NPs with well-defined shapes and monodisperse sizes. However, the amount of NPs results small and the cost quite expensive. Moreover, the dispersion of NPs for the integration into devices represent and additional challenge due to the inherent tendency of NPs to agglomerate.

An interesting approach to obtain metallic NPs dispersed onto a dielectric substrate overcoming the abovementioned limitations consists in the deposition of metallic films followed by a subsequent thermal annealing ${ }^{17,18,19}$. The difference in the thermal expansion coefficient between the substrate and the metal induces interfacial stresses that relax by breaking the continuous film into NPs during the cool-down process ${ }^{20}$. The size and shape of the NPs depends on the metallic thin film thickness, annealing conditions and substrate characteristics. This procedure is particularly interesting for noble metals as gold, since their chemical stability prevents oxidation during the annealing process. Several works reported on the fabrication of Au NPs supported onto a dielectric substrate using this method ${ }^{18,21,22,23}$. For the case of Au NPs, a univocal correlation between the deposition and annealing conditions with the NP morphology and the optical properties has been observed, in particular with SPR excitation $^{23}$.

However, the study of the SPR in the NPs was performed in the far field regime recording the average absorption spectra over macroscopic regions. A key element of SPR is the capability to concentrate and amplify locally the light raising up huge gradients of electromagnetic field 
in nanometric distances $1^{, 5,16}$. Therefore, a proper analysis of the optical properties of these nanostructures requires a study of the SPR excitation with spatial nanometric resolution. Such study can be carried out with a Scanning Near Field Microscope (SNOM) ${ }^{24}$. In this work we address the study of the optical properties of Au NPs supported onto glass substrates by means of SNOM.

\section{II - EXPERIMENTAL}

\section{A. Sample preparation}

Au NPs supported onto soda-lime glass cover-slides were prepared as previously described in reference 23. Au films were deposited on clean sodalime glass substrates in a vacuum chamber by thermal evaporation of $\mathrm{Au}$ wires placed on a tungsten filament. During the deposition, the base pressure in the chamber was $10^{-6}$ torr. The films were deposited at a rate of $0.02 \mathrm{~nm} \mathrm{~s}^{-1}$ measured with a quartz microbalance, and the nominal thickness was $10 \mathrm{~nm}$. The final deposited film was $10 \pm 0.2 \mathrm{~nm}$, measured with a stylus profilometer. After deposition, the resulting $\mathrm{Au}$ thin films were annealed in air employing a convection oven at $300^{\circ} \mathrm{C}, 400^{\circ} \mathrm{C}$ and $500^{\circ} \mathrm{C}$. The annealing consisted on an initial temperature ramp of 2 hours, 3 hours at the target temperature and a slow cooling down, with a ramp of approximately 5 ${ }^{\circ} \mathrm{C} / \mathrm{min}$.

\section{B. Morphological characterization}

Topographic information of the Au thin films was acquired with an Atomic Force Microscope (AFM) in air, with a silicon nitride tip in non-contact mode. Optical absorption spectra were recorded using a Shimadzu 3100 spectrophotometer. Local optical and topographical characterization of the samples was carried out using a Veeco Aurora III scanning near-field optical microscope. The used SNOM probes were aluminum-coated tapered optical fibers with nominal apertures of 50-80nm for SNOM and 120-150nm apertures for SNOMspectroscopy. The images were taken in illumination-transmission mode at a constant distance from the surface. The tip-surface distance control was performed with an electronic feedback mechanism based on a piezoelectric quartz tuning-fork acting as a shear-force sensor ${ }^{25}$. Two light sources were used, a 488nm air-cooled Ar-Ion laser for conventional SNOM, and a highpower white LED for the SNOM-spectroscopy, spanning from 400 to $700 \mathrm{~nm}$. The transmitted 
light was collected in the far-field with a microscope objective (0.65NA, infinity corrected) and directed to a photomultiplier tube (PMT) or to a spectrometer. The spectrometer used was a $0.5 \mathrm{~m}$ monochromator with a liquid nitrogen-cooled CCD and a $3001 / \mathrm{mm}$ grating with blaze at $500 \mathrm{~nm}$. The spectrometer was electronically synchronized with the SNOM to acquire single spectrum at every point of the image with an integration time of $200 \mathrm{~ms}$.

Figure 1 shows AFM images of samples annealed at temperatures ranging from $300^{\circ} \mathrm{C}$ to $500^{\circ} \mathrm{C}$. The sample annealed at $300^{\circ} \mathrm{C}$ presents island of irregular shapes with lengths up to $1 \mu \mathrm{m}$. For the sample annealed at $400^{\circ} \mathrm{C}$ we observe a similar behavior, but the length of the islands becomes shorter, with maximum lengths of approximately 500nm, and more rounded islands of $200-500 \mathrm{~nm}$ diameters appear. When the films are annealed at $500^{\circ} \mathrm{C}$ we can see mainly two types of islands: a family of rounded islands with diameters ranging between 90 and 400nm and another family of smaller particles with diameters of 40nm and less; while these kind small particles are present in all samples, it is at the sample annealed at a higher temperature at which they are present at most spaces between the bigger isluniformly throughout the whole sample.
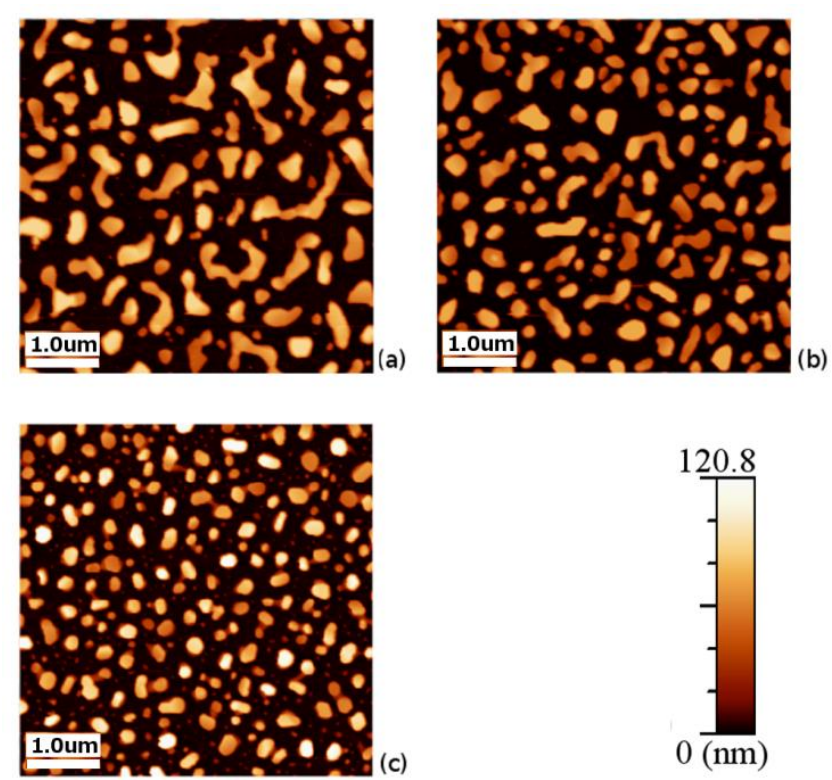

FIG.1. (Color online) Non-contact AFM images of the samples annealed at (a) $300^{\circ} \mathrm{C}$, (b) $400^{\circ} \mathrm{C}$ and (c) $500^{\circ} \mathrm{C}$.

The histograms of the island area for the three samples in Figure 2 show the island area as a function of the annealing temperature and confirms the existence of this family of smaller gold nanoparticles. For the three annealing temperatures, the average height of the islands is 
the same, around $60 \mathrm{~nm}$. The mean value for the islands area is $79900 \mathrm{~nm}^{2}$ for the sample annealed at $300^{\circ} \mathrm{C}, 53200 \mathrm{~nm}^{2}$ for the one annealed at $400^{\circ} \mathrm{C}$ and $20800 \mathrm{~nm}^{2}$ for the sample annealed at $500^{\circ} \mathrm{C}$ (since the last are rounded, we can say that they have a mean radius of 90$100 \mathrm{~nm})$. The area covered in gold is approximately the same for the three samples, around $36 \%$ of the surface. These results show that as the annealing temperature increases the islands become smaller, in agreement with previous results ${ }^{23}$.

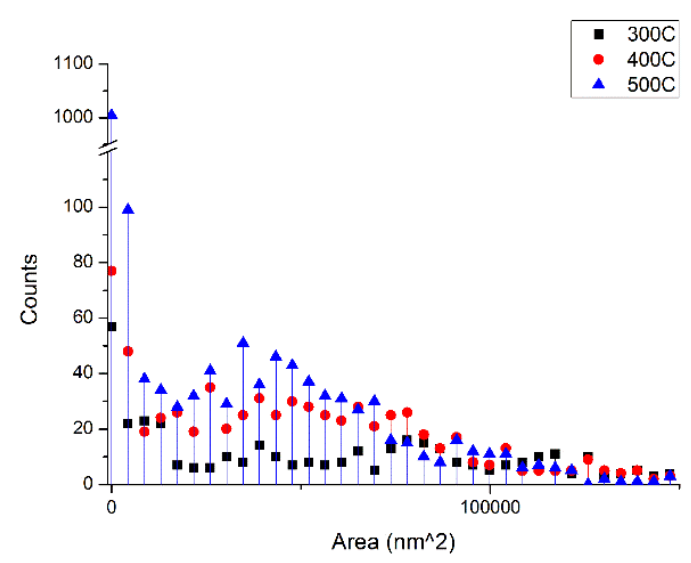

FIG.2. (Color online) Plot of the island area histograms for the samples annealed at $300^{\circ} \mathrm{C}$ (black squares), $400^{\circ} \mathrm{C}$ (red circles) and $500^{\circ} \mathrm{C}$ (blue triangles). The three plots represent the number of islands of different sizes in a $10 \mu \mathrm{m} \times 10 \mu \mathrm{mx}$ area of the sample. The size dispersion and the average size of the islands gets narrower for higher annealing temperatures. A family of very small particles appears when annealing at $500^{\circ} \mathrm{C}$. The histogram for the samples annealed at $300^{\circ} \mathrm{C}$ extends longer in the $\mathrm{X}$ axis, but we decided to cut it to make it easier to compare the three samples.

\section{Optical characterization}

The optical absorption spectra of the samples are presented in figure 3 together with the absorption of a bare substrate. All the spectra exhibit a sharp absorption edge at about 365 $\mathrm{nm}$, due to the soda-lime glass substrate and a band at about $450 \mathrm{~nm}$ that correspond to $\mathrm{Au}$ interband transitions which is characteristic of $\mathrm{Au}$ in both bulk and nanometric sizes ${ }^{5}$. In addition, the sample annealed at 500C exhibits a well-defined absorption band centered at 630 $\mathrm{nm}$ that corresponds to SPR excitation for rounded $\mathrm{Au}$ NPs as those observed in the corresponding AFM image (fig. 1c). On the contrary, the samples with non-rounded NPs (those annealed at 300C and 400C) exhibit a much wider absorption band that extends in the IR. 


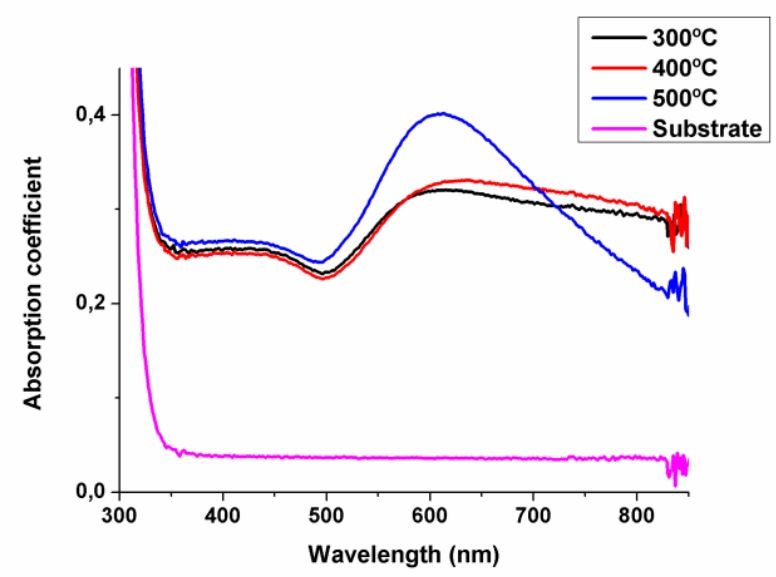

FIG.3. (Color online) Absorption spectra of Au thin films annealed at $300^{\circ} \mathrm{C}, 400^{\circ} \mathrm{C}$ and $500^{\circ} \mathrm{C}$. The sample annealed at $500^{\circ} \mathrm{C}$ shows a broad plasmonic peak centered at about $630 \mathrm{~nm}$ while the other two show a flat absorption band extending into the infrared region.

Figure 4 presents SNOM images for the different samples obtained in transmission mode using $488 \mathrm{~nm}$ light. The spatial distribution of transmittance resembles the topography of the samples, presenting opaque regions with sizes very similar to those of the nanoislands. For the used wavelength $(488 \mathrm{~nm})$, the absorption is due to $\mathrm{Au}$ interband transitions (particularly $3 \mathrm{~d}$ $5 \mathrm{~d}$ transitions). The probability of these transitions is independent of the size of the particle. Thus, the absorption at this wavelength is determined just by the number of $\mathrm{Au}$ atoms that are present in the beam path, and the image reproduces the topography of the sample.

In order to distinguish these optical properties due to interband transition from those related to excitation of SPR, we measured local absorption spectra at each point of the sample. To this purpose, we illuminated the sample with white light and recorded the absorption spectra point by point while scanning the sample with the SNOM tip. This allows the reconstruction of an optical absorption image by taking slices at different wavelengths within the emission range of the LED. 

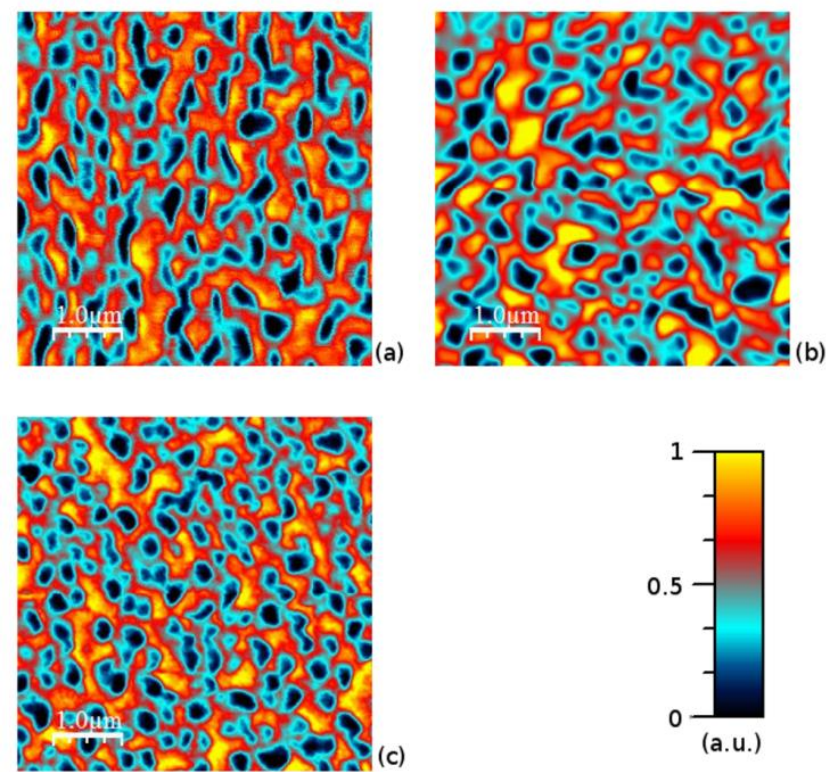

FIG.4. (Color online) SNOM images in transmittance (a.u.) at $488 \mathrm{~nm}$ for the samples obtained at (a) $300^{\circ} \mathrm{C}$, (b) $400^{\circ} \mathrm{C}$ and (c) $500^{\circ} \mathrm{C}$. The colors are scaled to the maximum and minimum transmittance recorded for each different sample.

Figure 5 presents topography and absorption images at different wavelengths for the sample annealed at 300C. The quality of the topography image when acquired with the SNOM is poorer than that of the AFM images presented in figure 1. This is a consequence of the SNOM tip radius (in the order of hundreds of nanometers). The SNOM tips employed are optimized for light excitation/collection and not for topography. However, the resolution is good enough to determine the position of the island, so we can correlate the optical measurement with the presence of NPs.
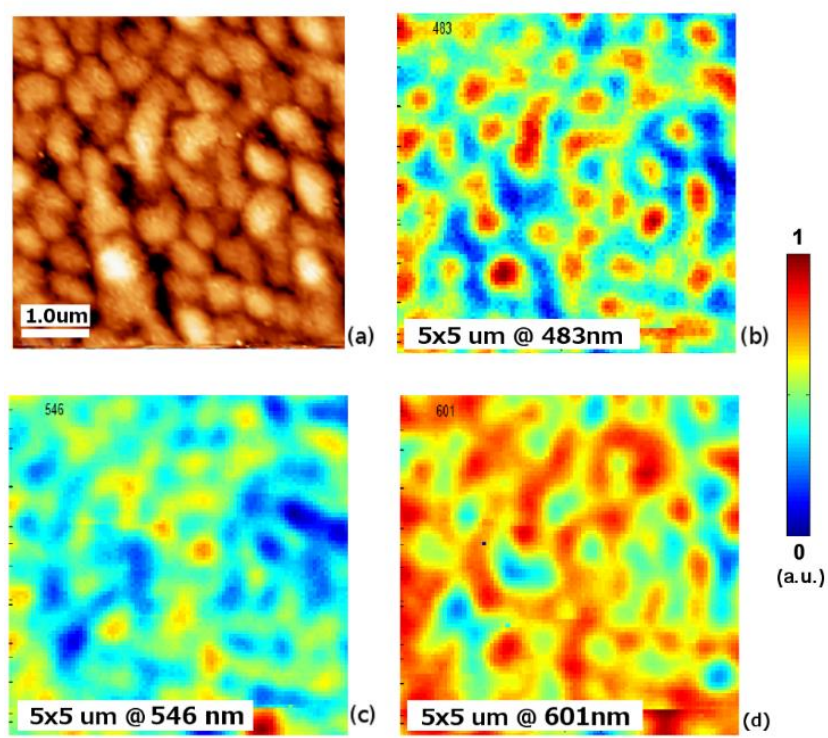
FIG.5. (Color online) Shear force topography (a) of the sample annealed at $300^{\circ} \mathrm{C}$ and the corresponding spectral slices at the representative wavelengths of $483 \mathrm{~nm}$ (b), $586 \mathrm{~nm}$ (c) and $601 \mathrm{~nm}$ (d). The spectral slices show the relative absorption at each wavelength, with 0 meaning the absorption of the glass substrate at the corresponding wavelength and 1 the maximum absorption recorded for each wavelength in the region analyzed.

The absorption image at $483 \mathrm{~nm}$ shown in figure $5 \mathrm{~b}$ presents a contrast that reproduces the spatial distribution of the NPs over the substrate as above discussed. The spatial distribution of absorption regions for $546 \mathrm{~nm}$ results completely different compared to that corresponding to $601 \mathrm{~nm}$ and neither of them reproduce exactly the topography of the surface. However, for this sample, the macroscopic absorption coefficient at these wavelengths resulted about identical (see figure 3).

Figure 6 shows three local spectra taken with the SNOM tip placed on top of three different islands with different shapes and sizes. These spectra differ from the far field spectrum in figure 3 because instead of showing an almost flat region for wavelengths over 580nm they show three well defined peaks centered at different wavelengths. The position of the peak depends on the size of the island. This difference is consequence of the wide distribution of NPs shapes and sizes that lead to a dispersion of SPR absorption bands. Thus, we may conclude that the flat profile of the macroscopic absorption spectrum is due to a contribution from different NPs, each one exhibiting absorption bands at different wavelengths.

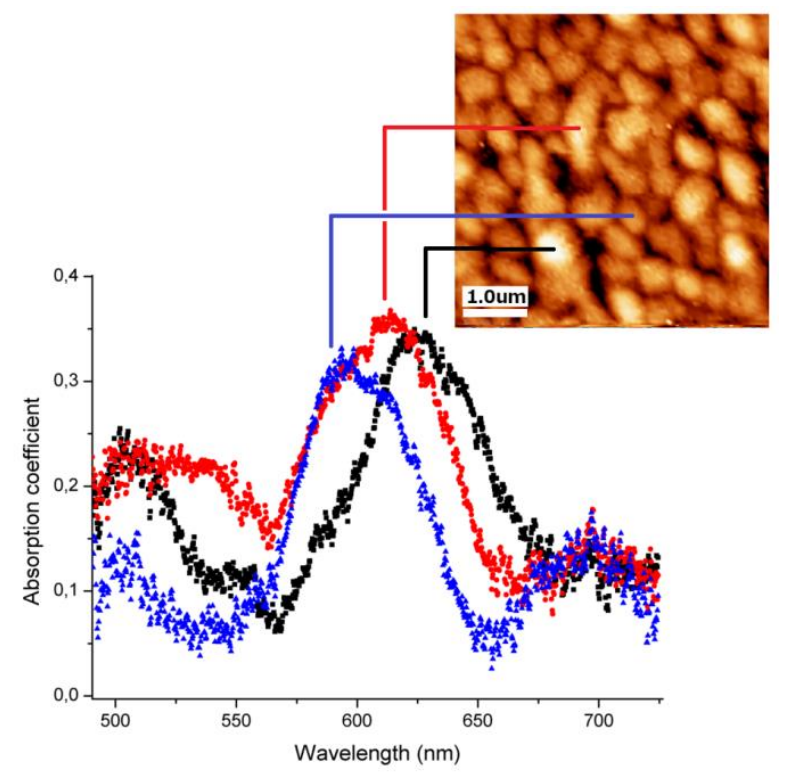

FIG.6. (Color online) Absorption spectra at the center of three individual nanoislands. The absorption peak shifts to longer wavelengths with increasing island size. 
Figure 7 shows another set of SNOM spectral slices of the absorption of the gold nanoislands, this tim for the $500^{\circ} \mathrm{C}$ sample, in which the size and shape dispersion is smaller. Also, this sample presents a big amount of smaller particles disseminated across the whole sample. The image taken at $483 \mathrm{~nm}$ (fig $7 \mathrm{~b}$ ) has a similar behavior compared to the equivalent image for the sample annealed at $300^{\circ} \mathrm{C}$ (fig $5 \mathrm{~b}$ ), with the absorption localized in the islands and thus reproducing the topography. The slice at $601 \mathrm{~nm}$ (fig $7 \mathrm{~d}$ ) also behaves the same way when compared to the sample annealed at $300^{\circ} \mathrm{C}$.

The main difference between the two samples appears at the slice taken at 546nm, where the sample annealed at $500^{\circ} \mathrm{C}$ shows clearly more absorptive regions than the previous sample. We attribute this affect to the presence of the family of smaller particles, which can be playing an important role in the plasmonic absorption at short wavelengths.
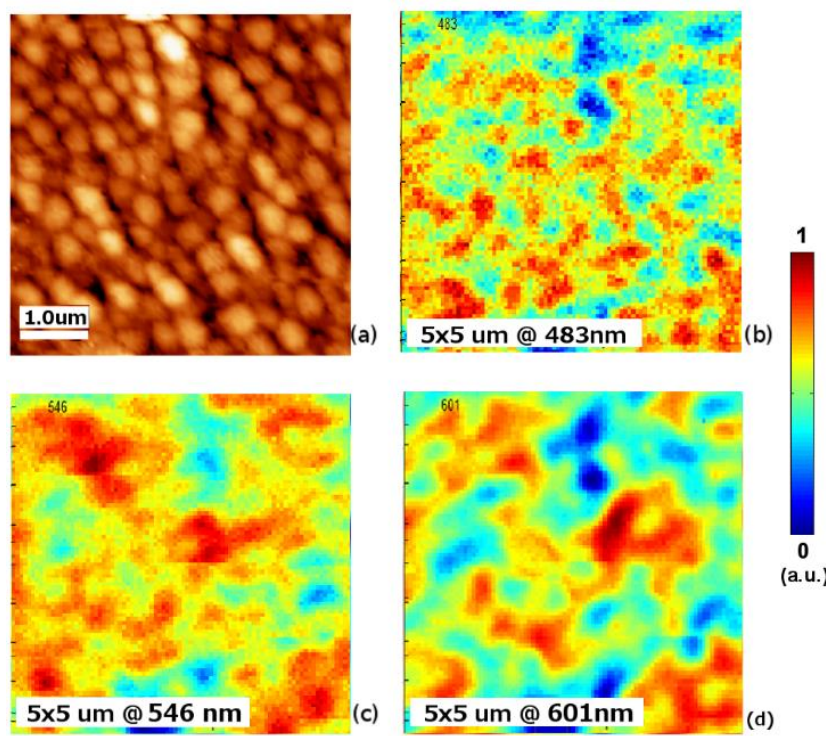

FIG.7. (Color online) Shear force topography (a) of the sample annealed at $500^{\circ} \mathrm{C}$ and the corresponding spectral slices at the representative wavelengths of $483 \mathrm{~nm}$ (b), $586 \mathrm{~nm}$ (c) and $601 \mathrm{~nm}$ (d). The spectral slices show the relative absorption at each wavelength, with 0 meaning the absorption of the glass substrate at the corresponding wavelength and 1 the maximum absorption recorded for each wavelength in the region analyzed.

\section{SUMMARY}

In summary, we showed here the advantages of the use of SNOM-spectroscopy in the analysis of plasmonic gold NPs ensembles. In the case of well isolated NPs with a small size and shape dispersion, the macroscopic optical absorption spectrum results similar to that of the 
individual NPs. On the contrary, when the NPs spatial distribution and morphology is not homogenous, the macroscopic optical properties are different to those of the individual NPs. In those conditions, an analysis in the near field region using a SNOM leads into a proper understanding of the ensemble optical properties. For the case of Au NPs obtained by annealing of thin films, SNOM analysis demonstrated here that the macroscopic optical properties of the system does not resemble that of the individual NPs but they are controlled by the particles morphology, that can be tuned through the preparation parameters. Preparation of samples with less dispersion on size, shape and inter-particle distances could lead to a better understanding of the related effects, giving us the possibility to further characterize this tipe of irregular samples.

\section{References}

${ }^{1}$ U. Kreibig and M. Vollmer, Optical Properties of Metal Clusters, Springer Series in Material Science Vol. 25 (Springer-Verlag, Berlin. 1995).

${ }^{2}$ M. L. Brongersma and P. G. Kik, Surface Plasmon Nanophotonics (Springer-Verlag, Berlin. 1988).

${ }^{3}$ S. A. Maier, Plasmonics (Springer-Verlag, Berlin. 2006).

${ }^{4}$ H. A. Atwater and A. Polman, Nature Materials 9, 205 (2010).

${ }^{5}$ M. A. Garcia, Journal of Physics D: Applied Physics 44, 28 (2011) 283001

${ }^{6}$ W. Fritzsche and T. A. Taton, Nanotechnology 14, R63 (2003).

${ }^{7}$ M. Hu, J. Chen, Z. Y. Li, L. Au, G. V. Hartland, X. Li, M. Marquez and Y. Xia, Chem. Soc. Rev 35, 1084 (2006).

${ }^{8}$ S. Eustis and M. A. El-Sayed, Chem Rev. Soc. 35209 (2006).

${ }^{9}$ A. Tiwari and S. Pilla, editors, Recent Developments in Bio-Nanocomposites for Biomedical Applications (Novascience Publishers INC, Hauppauge NY, 2010).

${ }^{10}$ S. Pillai, K. R. Catchpole, T. Trupke and M. A. Green, J. Appl. Phys. 101093105 (2007).

${ }^{11}$ P. Matheu, S. H. Lim, D. Derkacs, C. McPheeters and E. T. Yu, App. Phys. Lett. 93113108 (2008)

${ }^{12}$ R. Narayanan and M. A. El-Sayed, J. Phys. Chem. B 10912663 (2005).

${ }^{13}$ E. M. Larsson, C. Langhammer, I. Zorić and B. Kasemo, Science 3261091 (2009)

${ }^{14}$ K. Awazu, M. Fujimaki, C. Rockstuhl, J. Tominaga, H. Murakami, Y. Ohki, N. Yoshida, T. Watanabe, J. Am. Chem. Soc. 1301676 (2008).

${ }^{15}$ P. Zijlstra, J. W. M. Chon and M. Gu, Nature 459410 (2009).

${ }^{16}$ J. A. Schuller, E. S. Barnard, W. Cai, Y. C. Jun, J. S. White and M. L. Brongersma, Nature Materials 9193 (2010).

${ }^{17}$ A. E. B. Presland, G. L. Price, and D. L. Trimm, Surf. Sci. 29, 424 (1972)

${ }^{18}$ S. K. Sharma and J. Spitz, Thin Solid Films 65, 339 (1980).

${ }^{19}$ M. S. Rahman Khan, Bull. Mater. Sci. 9, 55 (1987).

${ }^{20}$ L. B. Freund and S. Suresh, Thin Film Materials: Stress, Defect Formation and Surface Evolution (Cambridge University Press, Cambridge. 2003). 
${ }^{21}$ J. A. Thornton and D. W. Hoffman, Thin Solid Films 171, 5 (1989).

${ }^{22}$ I. Doron-Mor, Z. Barkay, N. Filip-Granit, A. Vaskevich, and I. Rubenstein, Chem. Mater. 16, 3476 (2004)

${ }^{23}$ A. Serrano, O. Rodríguez de la Fuente, and M. A. García, J. Appl. Phys. 108074303 (2010).

${ }^{24}$ B. Hetch, H. Bielefeldt, L. Novotny, Y. Inouye, D.W. Pohl. Phys. Rev. Lett. 77, 9, 18891892 (1996)

${ }^{25}$ K. Karrai and R. D. Grober. Appl. Phys. Lett. 66, 1842 (1995) 